\title{
Vehicle route optimization of Centrally Dynamic Route Guidance Systems
}

\author{
H. Zhang ${ }^{1}$, J. Q. Sun ${ }^{1}$, Q. J. Hui ${ }^{2} \&$ J. Guo ${ }^{2}$ \\ ${ }^{1}$ College of Traffic Engineering and Logistic, \\ Dalian Maritime University, P. R. China \\ ${ }^{2}$ Public Security Bureau of Baicheng City, Baicheng, P. R. China
}

\begin{abstract}
Because of severe traffic jams and congestion, nowadays every country applies modern science and technology to solve these problems. This paper will utilize the real-time traffic volume data, the relationship between traffic volume and travel time and the incremental-load short-distance optimization algorithm to research the vehicle route optimization technique of Centrally Dynamic Route Guidance Systems which is one branch of Urban Traffic Flow Guidance Systems (UTFGS, the core of Intelligent Transportation Systems).

Keywords: ITS, UTFGS, CDRGS, traffic flow information, incremental-load short-distance optimization.
\end{abstract}

\section{Introduction}

Nowadays, the ever-fast progress of the world's economy stimulates the traffic requirement. Because of the continued increase in traffic volume and the limited construction of highway facilities in urban, intercity and rural areas, transportation has become the 'bottleneck' of modern cities where severe traffic congestion occurs frequently. To some degree it restricts people from going out. For example, in the USA the loss caused by traffic congestion and delay is estimated to be more than 100 billions US dollars [1,2,12]. If these problems are not solved, they will continue to be an obstacle to the progress of the whole of society. In order to improve the management of urban traffic, many countries, including China, apply modern science and technologies, including network 
system control, Intelligent Transportation System (ITS) etc. ITS is known as an effective way of managing urban traffic. Consequently there are three typical systems of ITS in Europe, USA and Japan.

ITS includes electronic surveillance, communications, and traffic analysis and control technologies, which bring benefits to users and managers. So, ITS can improve safety, effectiveness and the environment of the transportation system. Urban Traffic Flow Guidance System (UTFGS) [3, 4, 8-10], as a major part of ITS, can solve existing traffic problems effectively. As a part of UTFGS, the Centrally Dynamic Route Guidance Systems (CDRGS) uses dynamic traffic flow information to provide route guidance to the vehicle's requirements. There are many short-distance optimization algorithms, such as Chaotic Neural Network algorithm, Ant algorithm, etc. So, in this paper we will introduce the incremental-load short-distance optimization algorithm which is based on the real-time traffic flow information. Although it is an equilibrium algorithm, this method does not yield an equilibrium solution [17].

\section{Basic idea of the incremental-load short-distance optimization algorithm}

It is known that travel costs, which refer to travel time or travel distance, are an important criterion to judge whether the route guidance is optimized. In this paper the incremental-load short-distance optimization algorithm has the integrative feature of traffic guidance and control and chooses the optimized travel route for the customer.

According to traffic theory, there is biquadratic relationship between link travel time and link traffic volume [6]:

$$
t=t_{0}\left[1+0.15(V / C)^{4}\right]
$$

Then, considering a single link, link travel time increases as link traffic volume increases. As for several links, the link time-volume curve will possibly intercross, as shown in fig. 1(a), (b).

So, the basic idea of the incremental-load short-distance optimization algorithm is [5-11, 16]: firstly, load the traffic volume of all links stepwise into the corresponding link based on a given proportion and utilize the link-block function to calculate link travel time. Then, judge whether the link time-volume curves intercross from fig. 1 to provide real-time optimized route guidance: if they are intercrossed, the optimized route must contain the links in which travel time is lower than the crossing-point $M$; if they do not intersect, the optimized route contains links which have the shortest travel time. Repeat the computing procedure described above to modify the optimized route, until the traffic volume data of all the links in the network are exhibited, then the final optimized route can be obtained. 


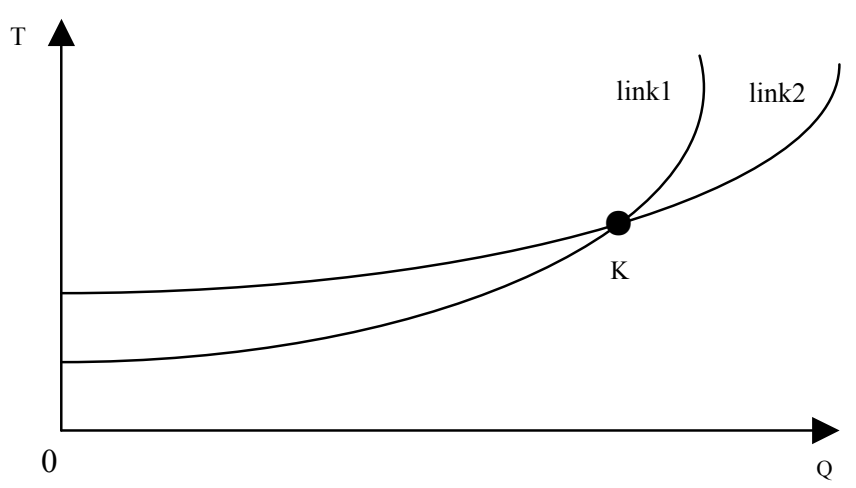

(a)

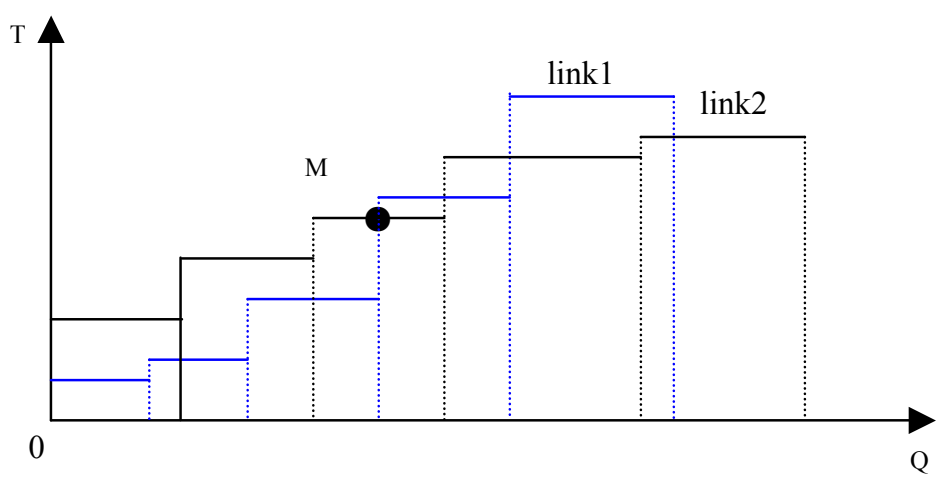

(b)

Figure 1: The relation between link traffic volume and travel time.

\section{Real-time traffic flow information prediction}

In this paper we use three statistical methods [11-15] and make use of the data gained from 28 detectors at the different intersections in Chang Chun city. The 28 detectors are shown as follows:

1 PA-CQR: N 1001; 2 PA-CQR: S 1012; 3 PA-CQR: W 1013; 4 PA-CQR:

E 1014; 5 LR-CS: W 3021; 6 LR-CS: E 3026; 7 LR-CS: S 3027; 8 LR-CS: N 3028; 9 PA-LR: S 3002; 10 PA-LR: N 3007; 11 PA-LR: W 3011; 12 PA-LR: E 3012; 13 XAR-GMR: E 2001; 14 XAR-GMR: W 2008; 15 LR-CS: W 3023; 16 LR-CS: E 3024; 17 LR-CS: S 3029; 18 LR-CS: N 3030; 19 PA-JSR: S 3001; 20 PA-JSR: N 3008; 21 PA-JSR: W 3009; 22 PA-JSR: E 3010; 23 LR-PYS: E 3015; 24 LR-DJR: E 3016; 25 LR-PYS: 
W 3018; 26 LR-DJR: S 3019; 27 LR-XMS: E 3025; 28 LR-XMS: W 3022.

PA- People Avenue, CQR- Chong Qing Road, LR- Liberation Road, CR- Comrade Street, XAR- Xi An Road, GMR- Guang Ming Road, LR- Liberation Road, CS- Construction Street,JSR- Jin Shui Road, DJ- DaJing Road, XMS- Xin Min Street. E-east, S-south, W-west, N-north.

\subsection{Cluster analysis method}

The parameters of system clusters are shown in Table 1. The system cluster result is shown in fig. 2.

In this example, we use the traffic volume of the 6th detector to predict the traffic volume at the east entrance of the Liberation Road - PingYang Street intersection. The equation of regression is:

$$
Q_{y}=41.58+0.73 Q_{x}
$$

In this equation $Q_{y}$ indicates the traffic volume that will be predicted and $Q_{x}$ indicates the traffic volume that is detected by the detector.

\subsection{Principal-factors analysis method}

The linear regression is shown as follows:

$$
\begin{gathered}
y=\left(-2.97+1.01 Z_{1}+0.63 Z_{2}-1.61 Z_{3}-0.6 Z_{4}-0.09 Z_{5}\right. \\
\left.+0.55 Z_{6}-0.35 Z_{7}\right) \cdot 10^{-5}
\end{gathered}
$$

Table 1: The parameters of system clusters.

\begin{tabular}{|c|c|c|c|c|c|c|c|}
\hline No. & $\begin{array}{c}\text { Similarity } \\
\text { Coefficient }\end{array}$ & Row & Column & No. & $\begin{array}{c}\text { Similarity } \\
\text { Coefficient }\end{array}$ & Row & Column \\
\hline 1 & 0.9910 & 24 & 23 & 2 & 0.9882 & 17 & 12 \\
\hline 3 & 0.9875 & 16 & 12 & 4 & 0.9808 & 15 & 12 \\
\hline 5 & 0.9772 & 18 & 12 & 6 & 0.9759 & 3 & 1 \\
\hline 7 & 0.9743 & 4 & 1 & 8 & 0.9739 & 6 & 2 \\
\hline 9 & 0.9706 & 8 & 1 & 10 & 0.9691 & 7 & 2 \\
\hline 11 & 0.9683 & 11 & 10 & 12 & 0.9667 & 18 & 10 \\
\hline 13 & 0.9606 & 18 & 9 & 14 & 0.9548 & 7 & 1 \\
\hline 15 & 0.9527 & 18 & 1 & 16 & 0.9523 & 26 & 23 \\
\hline 17 & 0.9497 & 25 & 23 & 18 & 0.9484 & 22 & 1 \\
\hline 19 & 0.9389 & 19 & 1 & 20 & 0.9369 & 14 & 1 \\
\hline 21 & 0.9007 & 5 & 1 & 22 & 0.8842 & 25 & 1 \\
\hline 23 & 0.8168 & 25 & 13 & 24 & 0.7869 & 28 & 13 \\
\hline 25 & 0.7463 & 27 & 13 & 26 & 0.6217 & 20 & 13 \\
\hline 27 & 0.5140 & 21 & 13 & & & & \\
\hline
\end{tabular}




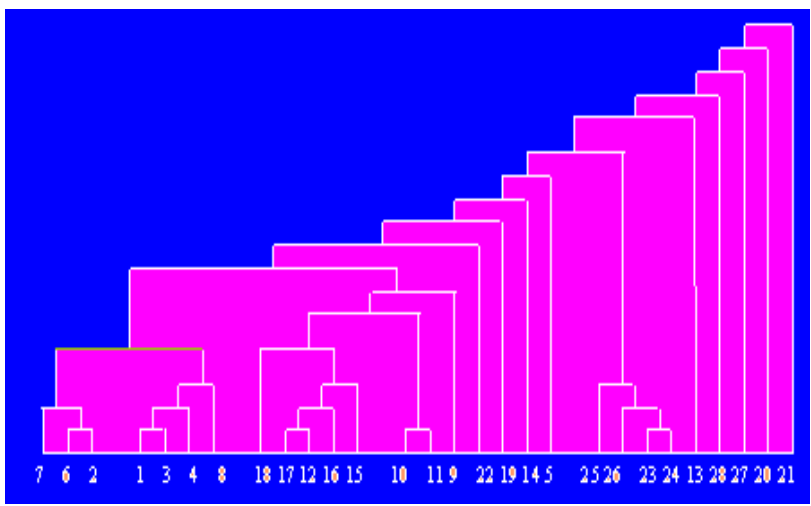

Figure 2: The pedigree chart of a system cluster.

In this example we adopt the $\boldsymbol{F}$ test method to judge the reliability of this model. The value of $\boldsymbol{F}$ is 27.9974 , and its theoretical value is 2.13 , and the multiple relative coefficient is 0.9535 .

Here we make use of the data gained from 25 detectors at the different intersections in Chang Chun city, and assume No. 1014 detector (the PA-CQR intersection east entrance) to be a non-detector intersection.

In this example, we obtained seven main-factors by calculation. The coefficients of these seven main-factors are as shown in Table 2.

\subsection{Stepwise regression method}

In this example, the number of samples is 26 , the number of variables is 24 , and only two variables were exhibited in the equation: No. 3019 and No. 1001. The linear regression equation is shown as follows:

$$
y=-3.78 \cdot 10^{-20}-0.11 x_{1}+0.32 x_{2}
$$

The $F$ test value is 7.7006 , its theoretical value is 2.13 , and the multiple relative coefficient is 0.9535 .

\section{Calculation of link-weight}

Based on the real-time traffic flow information, in CDRGS we must calculate the link-weight. The link-weight (which refers to the travel time between two intersections) is given by $[5-11,17]$ :

$$
T(i, j)=t(i, j)+d(i, j)
$$

where: $T[i, j]=$ link-weight;

$t[i, j]=$ link travel time;

$d[i, j]=$ average delay between intersection No. $i$ and intersection No. $j$. 
Table 2: $\quad$ Main-factors.

\begin{tabular}{|c|c|c|c|c|c|c|c|}
\hline$\frac{\text { Main-factors }}{\mathrm{n}}$ & $\mathrm{z}_{1}$ & $\mathrm{z}_{2}$ & $\mathrm{Z}_{3}$ & $\mathrm{Z}_{4}$ & $\mathrm{Z}_{5}$ & $\mathrm{Z}_{6}$ & $\mathrm{Z}_{7}$ \\
\hline 1 & 0.2134 & 0.0778 & -0.3162 & -0.0610 & 0.1048 & -0.0548 & 0.0465 \\
\hline 2 & 0.2131 & 0.0803 & -0.2948 & 0.0658 & 0.1234 & 0.1423 & -0.0725 \\
\hline 3 & 0.2145 & 0.0912 & -0.2875 & 0.0555 & 0.0783 & 0.0945 & -0.0132 \\
\hline 4 & 0.2159 & 0.1166 & -0.0190 & 0.1090 & -0.0225 & -0.0463 & -0.3068 \\
\hline 5 & 0.2223 & 0.0851 & -0.0956 & 0.0557 & 0.0374 & 0.0927 & -0.2399 \\
\hline 6 & 0.2095 & 0.1140 & -0.1587 & -0.1319 & 0.0391 & -0.2697 & -0.2283 \\
\hline 7 & 0.1998 & -0.3103 & -0.0156 & 0.0075 & -0.1724 & 0.0527 & -0.0472 \\
\hline 8 & 0.2031 & -0.2986 & 0.0469 & 0.0128 & -0.1037 & -0.0254 & 0.0513 \\
\hline 9 & 0.1995 & -0.3096 & 0.0515 & 0.0772 & -0.1202 & 0.0594 & 0.0403 \\
\hline 10 & 0.2061 & -0.2945 & 0.0061 & 0.0330 & -0.1123 & 0.0077 & 0.0458 \\
\hline 11 & 0.1762 & 0.2020 & -0.1030 & 0.5522 & -0.1343 & 0.1420 & 0.2156 \\
\hline 12 & 0.1945 & 0.1925 & -0.2585 & 0.2146 & 0.0715 & 0.1478 & 0.4383 \\
\hline 13 & 0.2079 & -0.2731 & -0.0153 & 0.0014 & -0.1055 & -0.0752 & -0.0360 \\
\hline 14 & 0.1230 & -0.2006 & 0.3175 & 0.3640 & 0.8145 & -0.1384 & -0.0994 \\
\hline 15 & 0.2060 & -0.2967 & -0.0053 & -0.0178 & -0.0710 & -0.0177 & -0.0004 \\
\hline 16 & 0.2053 & -0.2777 & 0.0154 & -0.0621 & -0.0828 & -0.0508 & 0.1391 \\
\hline 17 & 0.2095 & 0.1518 & 0.0071 & -0.2759 & 0.0500 & -0.0509 & -0.2092 \\
\hline 18 & 0.1574 & 0.1511 & 0.5091 & -0.1126 & -0.0442 & 0.6311 & -0.0505 \\
\hline 19 & 0.1858 & 0.0307 & 0.0837 & -0.4024 & 0.2673 & 0.1176 & 0.5210 \\
\hline 20 & 0.2099 & 0.1075 & -0.0636 & -0.3512 & 0.1103 & -0.1883 & 0.0268 \\
\hline 21 & 0.2136 & 0.1155 & 0.0160 & -0.0800 & 0.0309 & 0.2300 & -0.3303 \\
\hline 22 & 0.1853 & 0.2178 & 0.2680 & -0.0714 & -0.0518 & -0.4269 & 0.2323 \\
\hline 23 & 0.2208 & 0.1253 & 0.1035 & -0.0476 & -0.0550 & 0.0540 & -0.0946 \\
\hline 24 & 0.2064 & 0.1532 & 0.2321 & 0.0160 & -0.1202 & -0.0672 & 0.1391 \\
\hline 25 & 0.1710 & 0.2475 & 0.3239 & 0.2710 & -0.2792 & -0.3390 & -0.0334 \\
\hline
\end{tabular}

In the function, $t[i, j]$ is given by the link-block function and $d[i, j]$ is given by the modified Webster function.

\subsection{Calculation of link-block function $[5,6,11,17]$}

$$
U=\left\{\begin{array}{lr}
U_{0}(1-0.94 \cdot V / C), & V / C \leq 0.9 ; \\
U_{0} /(7.4 \cdot V / C), & V / C>0.9 .
\end{array}\right.
$$

where: $U_{0}$ - travel speed when traffic volume is zero $(\mathrm{km} / \mathrm{h})$

$$
U_{0}=r S \eta V_{0}
$$

where: $r=$ discount coefficient of bicycle;

$\eta=$ coefficient of lane width; 
$S=$ influencing coefficient of intersection;

$V_{0}=$ lane design speed

\subsection{Analysis of intersection delay}

If the saturation of the entry lane is less, the average delay of each entry lane is given by the modified Webster function [6,17]:

$$
d(i, j)=0.9 \times\left[\frac{T(1-\lambda)^{2}}{2(1-\lambda X)}+\frac{X^{2}}{2 Q(1-X)}\right] .
$$

where: $d(i, j)=$ average delay of the adjacent lane between intersection No.i and intersection No.j

$T=$ cycle length;

$\lambda=$ effective green time / cycle length on entry of lane;

$Q=$ traffic volume of lane;

$X=$ ratio of saturation $X=Q /(\lambda C)$.

If saturation of the inlet is large, the result gained by the Webster function is on the high side. The function below is proposed by the Handbook of Highway Capacity of America to estimate the delay on the entry lane:

$$
d=d_{1}+d_{2}
$$

where:

$$
\begin{gathered}
d_{1}=0.38 T \frac{(1-\lambda)^{2}}{(1-\lambda X)}, \\
d_{2}=173 X^{2}\left[(X-1)+\sqrt{(X-1)^{2}+16 X / S}\right] .
\end{gathered}
$$

where: $d_{1}=$ uniform delay;

$d_{2}=$ super-saturation delay (i.e., incremental delay caused by stochastic approach and additional delay caused by cycle invalidation); the meanings of other symbols are as described above.

In general, the Webster function is applied when the ratio of saturation is $\mathrm{X}=0$ - 0.66; the function proposed by Handbook of Highway Capacity of America is applied when ratio of saturation is $\mathrm{X}=0-1.20$. 


\section{Application}

This paper makes use of the data obtained from 25 detectors at the different intersections in Chang Chun city to perform travel route optimization and obtain satisfactory results. The final optimization route of CDRGS, which is selected by the incremental-load short-distance optimization algorithm, is shown in fig. 3.

\section{Conclusion}

In this paper, we adopt the Cluster Analysis Method, the Principal-component Analysis Method and the Stepwise Regression Method to predict traffic volume at non-detector intersections. Then, we make use of detected traffic volume data and predicted traffic volume data to perform network traffic flow guidance. So, we can achieve centrally dynamic route guidance and macro management of the whole urban network.

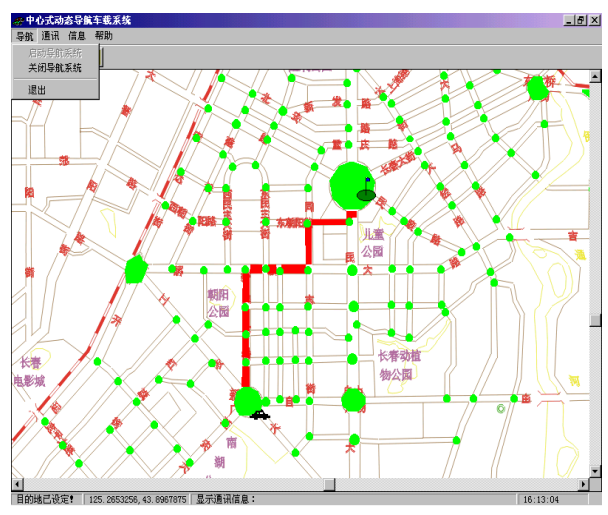

Figure 3: Travel route optimization.

\section{Acknowledgements}

This paper is supported NSFC (60602020) and the National Basic Research Program of China (2006CB705501).

\section{References}

[1] Z.S. Yang, Theory and Model of Urban Traffic Flow Guidance, People Transportation Publishing House, PK: 2000.1

[2] Y.L. Zhao, Vehicle Location and Navigation System, Publishing House of Electronics Industry, PK: Apr. 1999 
[3] Y.L. Gu, Research on Real-time Dynamic Traffic Flow Forecast in Traffic Flow Guidance Systems, [thesis for master degree], Jilin University of Technology, 1999

[4] H. Zhang, Research on Prediction Method of Traffic Information on Nondetector Intersection, [thesis for master degree], Jilin University of Technology, Mar. 2000

[5] FHWA. Traffic Assignment: Methods, Application and Products. U.S. Department of Transportation, 1973.

[6] W. Wang, J.Q. Xu, T. Yang, and X.H. Li, Urban Transportation Planning Theory and Application, Southeast University Publishing House, 1998

[7] Anthony Chen, Panatda Kasikitwiwat, and Zhaowang Ji. Solving the Overlapping Problem in Route Choice with Paired Combinatorial Logit Model. In Transportation Research Record 1852, TRB, National Research Council, China, 2003, pp.65-73.

[8] L.Y. Chu, Research on Theory and Application Technology of Dynamic Route Guidance System, [thesis for doctor degree], Jilin University of Technology, May. 1999

[9] D.S. Ma, The Travel Route Optimization and Implement Technology Research in DRGS, [thesis for master degree], Jilin University, Mar. 2002

[10] Daniel · L · Gerlough and Matthew - J · Huber, Traffic Flow Theory, Washington, D.C. 1975

[11] Z.S. Yang, H. Zhang, and Damachi B.B.U., Research on the method used in predicting the traffic volume on non-detector intersections, $9^{\text {th }}$ ITS 2000 , Chicago, U.S.A

[12] D.H. Wang, Z.S. Yang, and H. Zhang. Main-composition analysis used in dealing with traffic flow information. $2^{\text {nd }}$ ICTTS 2000

[13] H. Zhang and Z.S. Yang, Stepwise Analysis Used in Predicting Traffic Flow Information of Non-detector Intersections. Journal of Jilin University (Engineering) Vol.32 No.108, 2002

[14] D.H. Wang, Z.S. Yang, and H. Zhang. Cluster analysis used in dealing with traffic flow information. IEEE IVEC'99.

[15] J.L. Luo and Y. Xing, Analysis Method of Economic Statistic and Prediction, TsingHua University Publishing House, PK: 1987

[16] Blunden W.R., The Land-Use and Transportation System. Pergamon Press, 1984

[17] SHEFFI Y., Urban transportation network [M]. Massachusetts Institute of Technology, 1984. 